EGU2020-18067

https://doi.org/10.5194/egusphere-egu2020-18067

EGU General Assembly 2020

(c) Author(s) 2020. This work is distributed under

the Creative Commons Attribution 4.0 License.

\title{
Automated recognition and picking of foraminifera using the MiSo (microfossil sorter) prototype
}

Thibault de Garidel-Thoron, Ross Marchant, Martin Tetard, Michael Adebayo, and Yves Gally Aix Marseille Univ, CNRS, IRD, Coll France, INRAE, CEREGE, Aix-en-Provence, France (garidel@cerege.fr)

Recent progresses in image processing, and image recognition have paved the way for automated procedures to classify natural objects such as foraminifera. Foraminifera are among the most useful tracers in biostratigraphy and paleoceanography. Yet, the protocol used to extract and recognize the foraminifera has not changed since the mid- $18^{\text {th }}$ century: manual picking using a brush with a stereomicroscope.

Here we present the results we achieve by developing the MiSo - Microfossil Sorter - automaton, to automatically pick microfossils from the sediment coarse fraction. This automated system, built with ATG Technologies, is fully operational and works 24/7 at CEREGE. In this study, we will detail the basic workflow of the automaton, processing $~ 8000$ particles/day, and its ability to cope with the large morphological and structural variability of particles encountered in real, marginal to deep-sea sediments. We use convolutional neural networks adapted and trained on deep sea sediment samples to classify the coarse sediment particles, including planktonic and benthic foraminifera.

As a test case, we will compare paleoceanographic records generated by a micropaleontologist with the ones generated by our automaton: relative abundance, fragmentation rate, biometrical changes. We have studied two deep-sea cores from the equatorial Pacific to document past hydrographic changes in the late Quaternary, achieving millennial scale resolution through the last deglaciation. Using the automaton, we processed more than 500,000 foraminifera. The accuracy of recognition typically ranges around 85 to $95 \%$ depending of the morphoclasses and of the CNN used for the training. Morphoclass size probability density function and assemblages derived from the CNN will be compared to multi-proxy (micropaleontological and geochemical) records. We will discuss the ongoing applications of our workflow, from foraminifera to pteropods in deep sea sediments, and the recent updates of our system. 